\title{
Available Transfer Capability based Transmission Pricing Using optimal Power Flow Approach in Deregulated Electricity Market
}

\author{
Juily N. Sukalkar ${ }^{1}$, Dr. Sanjay Warkad ${ }^{2}$ \\ PG Student, Electrical Engineering, P.R. Pote (Patil) College of Engineering, Amravati, India ${ }^{1}$ \\ Assistant Professor, Electrical Engineering, P.R. Pote (Patil) College of Engineering, Amravati, India ${ }^{2}$
}

\begin{abstract}
Before deregulation there was only single entity to generate and distribute electricity in an area. Due to this monopoly, the price of electricity was on the higher side and also the quality of electricity was not satisfactory. This scenario changes after the deregulation of power industry. The competition arises in generation and distribution. But the transmission corridor remains the same for transferring the power. Every operator wants to maximize their profit. Due to this the number of transactions increases. This may cause congestion in transmission network. To avoid congestion, every operator should know the value of ATC before every transaction. The aim of this paper is to develop an Optimal Power Flow based approach to analyse electrical Transfer capability using Power Transfer Distribution Factor. Also Available Transfer Capability Based Transmission Pricing is evaluated. The above said methodology is applied on IEEE 30 bus system.
\end{abstract}

Keywords: Optimal Power flow, Transmission Pricing, Deregulation, Available Transfer capability.

\section{INTRODUCTION}

In most developing countries the electricity sector still follows the model of a centralized monopolistic utility. The pricing of energy is a mixture of elements, from social policy and employment considerations to economic spin-offs, basic equity considerations and sometimes political objectives. The primary challenge in developed countries in terms of market reform is usually to bring prices down to the competitive cost of service. However, in developing countries the challenges are to set prices high enough to cover the full cost of delivering the service and to ensure that payment is collected.

In electricity, LMP recognizes that this Locational Marginal Pricing (LMP) or Nodal pricing based on Short Run Marginal Costing (SRMC) principle. It is a market-pricing approach used to manage the efficient use of the transmission system when congestion occurs on the bulk power grid. Marginal price may vary at different times and locations based on transmission congestion. With LMP, market participants will know the price at hundreds of locations on the system. Under network constraints environment, electric grid congestion is a common phenomena. This can put restrictions on the transmission system; prevent the economic or least expensive supply of energy from serving the demand.

In this paper Optimal power flow based, Available Transfer capability computed electricity nodal pricing is proposed in a deregulated electricity market. Available transfer capability computations are essential for successful implementation of electric power deregulation

Where power producers and customers share a common transmission network. The available transfer capability indicates the amount which inter-area bulk power transfers can be increased without compromising system security. In this present open access or deregulated environment all the participants (producers and buyers of electrical energy) desire to produce or consume large amounts of energy and may force the transmission system to operate beyond one or more transfer limits. This kind of operation leads to congestion of the system. Therefore accurate determination of available transfer capability is essential to ensure the system security and reliability while serving a wide range of bilateral and multilateral power transactions.

\section{OPTIMAL POWER FLOW PROBLEM}

Optimal pricing method tries to maximize consumer net benefit, taking into account transmission losses and generator uncertainty. The main Objective of an optimal power flow is to minimize total operating cost of the system. In OPF, when the load is light, the cheapest generators are always the ones chosen to run first. As the load increases, more and more expensive generators will then be brought in. 
UGC Approved Journal

IJIREEICE

Vol. 5, Issue 7, July 2017

Thus, the operating cost plays a very important role in the solution of OPF. In all practical cases, the cost of generator i can be represented as a cubic function of real power generation expressed in $\$ / \mathrm{hr}$,

$$
C_{i}=a_{i}+b_{i} P_{g i}+c_{i} P_{g i}^{2}
$$

Where Pgi is the real power output of generator $i$, and $a, b$, and $c$ are the cost coefficients

Normally, the cost coefficients remain constant for a generator. Another important characteristic of a generator is the incremental cost, also known as marginal cost. It is a measure of how costly it will be to produce the next increment of power. The incremental cost can be obtained from the derivative of $\mathrm{Ci}$ of equation (1) with respect to $\mathrm{Pi}$,

$$
\frac{\partial c_{i}}{\partial P_{i}}=b_{i}+2 c_{i} P_{g i}
$$

Which is expressed in $\$ / M W h r$.

The transmission losses become a major factor in a large interconnected network whereby power is being transmitted over long distances. A common function to represent total system real power losses in terms of the total real power output is given by using formula

$$
P_{L}=\sum_{i=1}^{n g} \sum_{j=1}^{n g} P_{i} \mathrm{~B} \mathrm{ij} \mathbf{P} \mathrm{j}+\sum_{i=1}^{n g} \mathrm{~B} \text { oi Pi }+\mathrm{B} \text { oo }
$$

Where PL is the total real power losses, and Bij are the loss coefficients or B coefficients.

Optimal dispatch can be seen generally as a optimization problem. When solving optimization problem, there are two general types of constraints, which are equality and inequality constraints. Equality constraints are constraints that always need to be intensified.

The optimization problem can be solved using the Lagrange Multiplier method, and for simplicity, only the maximum and minimum real power limits are included as the inequality constraints.

Hence the total operating cost of all generators in a system is given by,

$$
C_{i}=\sum_{i=1}^{n g} C_{i}
$$

Where ng is the number of generator buses.

The total real power generation is then given by,

$$
\sum_{i=1}^{n g} P_{i}=P_{D}+P_{L}
$$

Where $\mathrm{Pi}_{(\min )} \leq \mathrm{Pi} \leq \mathrm{Pi}_{(\max )}$, PD is the total real power demand, and PL is the total system real power loss The Lagrange Multiplier can then be expressed as,

$$
L=C_{t}+\lambda\left(P_{D}+P_{L}-\sum_{i=1}^{n g} P i\right)+\sum_{i=1}^{n g} \pi_{i(\max )}\left(P_{i}-P_{i(\max )}\right)+\pi_{i(\min )}\left(P_{i}-P_{i(\min )}\right)
$$

Where the term second term is the equality constraint, while the last two terms are inequality constraints in equation. Note that both $\pi \mathrm{i}(\max )$ and $\pi \mathrm{i}(\min )$ are equal to zero if $\mathrm{Pi}(\min ) \leq \mathrm{Pi} \geq \mathrm{Pi}(\max )$, which means that the inequality constraints are inactive. The constraints will only be active when violated, which means $\mathrm{Pi}>\mathrm{Pi}(\max )$ or $\mathrm{Pi}<\mathrm{Pi}(\min )$. This is known as the Kuhn-Tucker necessary conditions of optimality, following the conditions below,

$$
\begin{aligned}
& \frac{\partial \ell}{\partial P_{i}}=0 \\
& \frac{\partial \ell}{\partial \lambda}=0
\end{aligned}
$$


UGC Approved Journal

IJIREEICE

International Journal of Innovative Research in Electrical, Electronics, Instrumentation and Control Engineering

ISO 3297:2007 Certified

Vol. 5, Issue 7, July 2017

$$
\begin{aligned}
& \frac{\partial \ell}{\partial \pi_{i(\min )}}=P_{i}-P_{i(\min )}=0 \\
& \frac{\partial \ell}{\partial \pi_{i(\max )}}=P_{i}-P_{i(\max )}=0
\end{aligned}
$$

The optimal solution can then be obtained by solving for the condition

$$
\frac{\partial \ell}{\partial \boldsymbol{P}_{i}}=\mathbf{O}
$$

\section{III.MARGINAL PRICING}

Marginal costing principals are very popular in the electricity sector due to its economic efficiency. The basic theory behind marginal costing can be explained using the following figure.

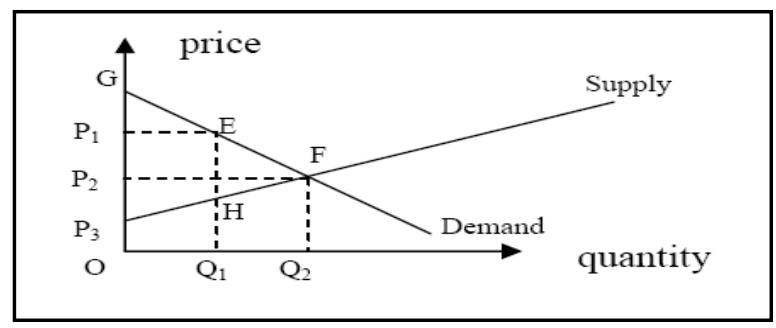

Fig 1: Typical Marginal Cost Curve

Marginal cost is the cost of supplying an extra unit of energy. In the above graph, the supply curve represents the marginal cost curve. The total benefit of consumption is the consumers' willingness to pay. For a price of $\mathrm{P} 1$ it is given by the area OQ1E P1. The cost of supplying the required quantity Q1 is given by the area OQ1H P3. Therefore the area P3HEG gives the net benefit. This indicates the maximum net benefit can be obtained when the price is $\mathrm{P} 2$. i.e. when the price equals marginal cost.

Electricity in the National Electricity Market can be either traded through retail or wholesale trading or even through contracts. All wholesale electricity must be traded through the spot market; generators are paid for the electricity they sell to the pool while retailers and wholesale end- users pay for the electricity they use from the pool. It is a process whereby prices for electricity are set and then settled. This pool is the way which short-term operation of the power system is centrally. In this spot market, generating companies can choose whether to commit their generators and make it available for dispatch.

\section{BACKGROUND OF ATC}

According to the definition of North American Electric Reliability Council (ATC) is a measure of the transfer capability remaining in the physical transmission network for future commercial activity over and above already committed uses. It is clear that ATC information is significant to secure operation deregulated power system as it reflects physical realities if power system such as costumer demand level, network paradigm, generation dispatch and transfer between neighbouring systems. Mathematically, ATC is obtained by subtracting transmission reliability margin, capacity benefit margin $(\mathrm{CBM})$ and existing transmission commitments from total transfer capability.

ATC $=$ TTC - TRM-CBM-ExistingTransmission Commitment

Where, (TTC): It is defined as the quantity of electric power that can be transferred over the interconnected transmission path reliably without violating the predefined set of conditions of the system

(TRM): It is defined as that amount of transmission transfer capability necessary to ensure that the interconnected transmission network is secure under a reasonable range of uncertainties in system conditions.

$(\mathrm{CBM})$ is defined as that amount of transmission transfer capability reserved by load serving entities to ensure access to generation from interconnected systems to meet generation reliability requirements.

\section{TEST RESULTS}

Here the ideas are demonstrated by using IEEE 30Bus system. This section Line Flow Analysis on different system condition is carried out and average base case Line Flow is obtained, which is given as below: 
UGC Approved Journal

IJIREEICE

Vol. 5, Issue 7, July 2017

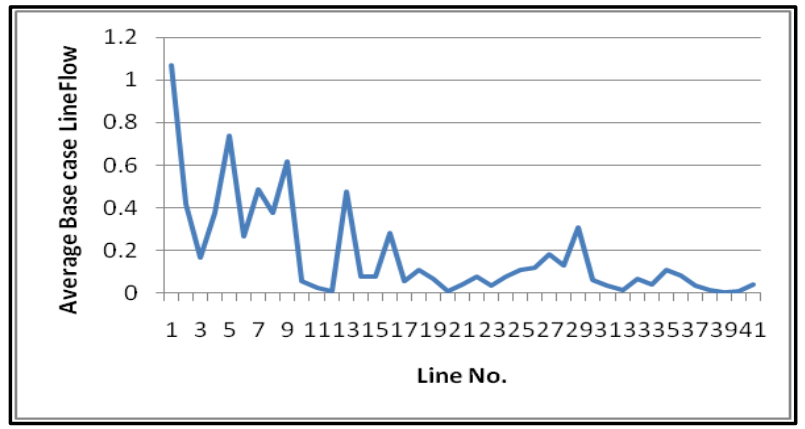

Fig 1: Average base case line flow

Here in this paper Optimal power flow algorithm is used for the evaluation of ATC. From above graphical analysis it is observed that there is congestion in line no. 1. So ATC is calculated for Line no. 1 only.

Here linear analysis of ATC is carried out that is by using power transfer distribution factor. Where PTDF is the fraction of the amount of a transaction from one area to another over a given transmission line It can be calculated as: $\mathrm{PTDF}=\frac{\text { Base Case Line Flow }- \text { Increased in Loading Case Line Flow }}{\text { Base Case Line Flow }(\mathrm{a} \rightarrow \mathrm{b})}$

Here PTDF is found to be:

\section{TABLE I PTDF CALCULATIONS}

\begin{tabular}{|l|l|l|}
\hline Interface & Max. MW Transfer & PTDF \\
\hline $1-2$ & 107.2 & 0.1732 \\
\hline
\end{tabular}

ATC between nodes (or areas) can be found by varying the amount of a transaction. The new flow in line 1 would be the sum of the original flow and the change. That new flow should be less than the line's thermal limit. Available Transfer capability for particular line can be calculated as:

$$
A T C=\frac{\text { Line l Thermal Limit }- \text { Original Flow on Line }}{\operatorname{PTDF}(\mathrm{a} \rightarrow \mathrm{b})}
$$

Here ATC based transmission pricing is find out by using optimal power flow method.

TABLE II ATC CALCULATIONS

\begin{tabular}{|l|l|}
\hline Interface & ATC \\
\hline $1-2$ & 102.7 \\
\hline
\end{tabular}

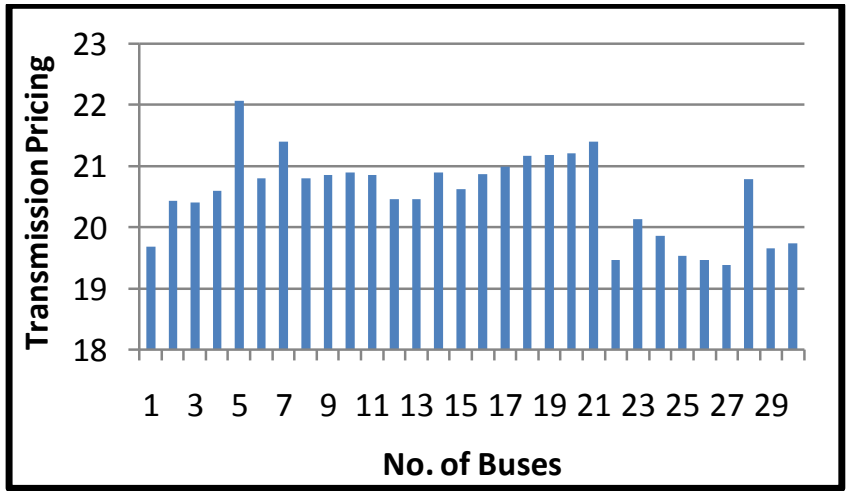

Fig 2: ATC Based Transmission Pricing

\section{VI.CONCLUSION}

This method demonstrated that the proper scheduling of generators by using OPF minimized the total system losses and therefore increased generators efficiencies. 
UGC Approved Journal

IJIREEICE

Vol. 5, Issue 7, July 2017

It shows that the OPF algorithm had solved the case more cost-efficiently. It is also realized that cheaper generators will have higher profit margins regardless of the spot prices. The impact of ATC on electricity nodal prices as shown in figure 2 concludes that Nodal prices are higher for congested transmission lines.

Also this study discussed the need of modeling electricity nodal prices for market participants and system operators under restructured electricity environment.

\section{REFERENCES}

[1] James Daniel Weber, Implementation of a Newton-Based Optimal Power Flow into a Power System Simulation Environment, A Thesis submitted to University of Illinois at Urbana-Champaign, pp. 1-102, 1997.

[2] Luonan Chen, Hideki Suzuki, Tsunehisa Wachi, and Yukihiro Shimura, Components of Nodal Prices for Electric Power Systems, IEEE Transactions on Power Systems, vol. 17, no.1, pp.41-49, February 2002

[3] Naresh K. Yadav, Prashant Sharma Available Transfer Capability Calculations Using DCPTDF Incorporating Multiple Line Contigincies and Generator Addition Under Deregulated Environment International Journal of Advanced Research in Electrical, Electronics and Instrumentation Engineering Vol. 2, Issue 6, June 2013

[4] M. Sivasatyanarayan, L. Rajasekhar Gou, J.Amarnath Available Transfer Capability (ATC) Under Deregulated Power Systems International Journal of Engineering and Management Research Volume-4, Issue-2, April-2014.

[5] Shabaan Mohemed, Mohamed Abdel Moneim Calculation of Available Transfer Capability of Transmission Network Including Static and Dynamic Security a thesis submitted to Hong kong University.

[6] K. Indhumathy, N.B. Rajesh, J. Jaya Priya Assessment of Available Transfer Capability (ATC) Using Linear Sensitivity Factors under Deregulated Environment International Journal of Engineering and Technology. 